\title{
Insulin supplementation attenuates cancer-induced cardiomyopathy and slows tumor disease progression
}

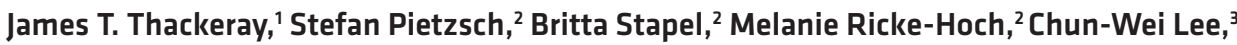 \\ Jens P. Bankstahl, ${ }^{1}$ Michaela Scherr, ${ }^{3}$ Jörg Heineke, ${ }^{2}$ Gesine Scharf, ${ }^{2}$ Arash Haghikia, ${ }^{2,4}$ \\ Frank M. Bengel, ${ }^{1}$ and Denise Hilfiker-Kleiner ${ }^{2}$ \\ 'Department of Nuclear Medicine, ${ }^{2}$ Division of Molecular Cardiology, Department of Cardiology and Angiology, and \\ ${ }^{3}$ Department of Hematology, Hemostasis, Oncology and Stem Cell Transplantation, Hannover Medical School, Hannover, \\ Germany. ${ }^{4}$ Department of Cardiology, Charité Universitätsmedizin Berlin (Campus Benjamin Franklin), Berlin, Germany.
}

Advanced cancer induces fundamental changes in metabolism and promotes cardiac atrophy and heart failure. We discovered systemic insulin deficiency in cachectic cancer patients. Similarly, mice with advanced B16F10 melanoma (B16F10-TM) or colon 26 carcinoma (C26-TM) displayed decreased systemic insulin associated with marked cardiac atrophy, metabolic impairment, and function. B16F10 and C26 tumors decrease systemic insulin via high glucose consumption, lowering pancreatic insulin production and producing insulin-degrading enzyme. As tumor cells consume glucose in an insulin-independent manner, they shift glucose away from cardiomyocytes. Since cardiomyocytes in both tumor models remained insulin responsive, low-dose insulin supplementation by subcutaneous implantation of insulin-releasing pellets improved cardiac glucose uptake, atrophy, and function, with no adverse side effects. In addition, by redirecting glucose to the heart in addition to other organs, the systemic insulin treatment lowered glucose usage by the tumor and thereby decreased tumor growth and volume. Insulin corrected the cancerinduced reduction in cardiac Akt activation and the subsequent overactivation of the proteasome and autophagy. Thus, cancer-induced systemic insulin depletion contributes to cardiac wasting and failure and may promote tumor growth. Low-dose insulin supplementation attenuates these processes and may be supportive in cardio-oncologic treatment concepts.

Authorship note: J.T. Thackeray, S. Pietzsch, and B. Stapel contributed equally as co-first authors. F.M. Bengel and D. Hilfiker-Kleiner contributed equally as co-senior authors.

Conflict of interest: The authors have declared that no conflict of interest exists.

Submitted: January 26, 2017

Accepted: April 11, 2017

Published: May 18, 2017

Reference information: JCI Insight. 2017;2(10):e93098 https://doi.org/10.1172/jci. insight.93098.

\section{Introduction}

Cancer and heart disease are major burdens on the healthcare system, in particular due to the aging population. Advanced stages of cancer are known to induce cachexia, a multifactorial disorder characterized by progressive breakdown of skeletal muscle and adipose tissue, regardless of nutritional support (1). Cachexia is thought to affect up to one-half of untreated cancer patients and has been implicated as principally responsible for up to $30 \%$ of oncologic mortality (2). Cachectic cancer patients exhibit symptoms of chronic heart failure, leading to speculation regarding common pathways for multiple cachectic conditions (3).

Little is known about the cause of heart failure in advanced cancer. Putative mechanisms implicate tumor-secreted factors, including proinflammatory cytokines (IL6, TNFA), proteolysis by ubiquitin-proteasome and autophagy-related lysosomal pathways, imbalance of catabolism and anabolism, and mitochondrial dysfunction $(4,5)$. Aggressive tumor growth may also interfere with normal cardiac metabolism (6). Depleted fat tissue and reduced fatty acid substrate metabolism in combination with impaired glucose tolerance, reduced insulin sensitivity, and aberrant pancreatic insulin release have been reported in cancer $(7,8)$. Epidemiologic evidence suggests a decrease in cancer risk in subjects treated with the insulin-sensitizing agent metformin (9) and higher rates of cancer in patients with metabolic diseases and impaired insulin signaling (10). Moreover, restoration of insulin sensitivity and modulation of fatty acid metabolism by the PPAR- $\gamma$ inhibitor pioglitazone prevents body mass wasting and improves survival in tumor-bearing rats (11). Independent of malignancy, dysfunctional cardiac insulin signaling has been implicated in heart failure, as evidenced, for example, in diabetic cardiomyopathy (12). 
Here, we hypothesized that aggressive tumor growth precipitates metabolic cardiac failure, not only by depletion of fatty acids, but also mainly by interfering with insulin signaling, which affects multiple processes beyond energy production, culminating in atrophy and contractile dysfunction. Indeed, we observed reduced systemic insulin levels in a clinical sample of patients with advanced tumors compared with healthy age- and gender-matched controls. To analyze whether cancer causes systemic insulin depletion and how this affects the heart, we generated two mouse tumor models using two different tumor cell types: B16F10 melanoma (B16F10-TM) and colon 26 carcinoma (C26-TM). Progressive growth of both B16F10 and C26 tumors lowered systemic insulin and, consequently, impaired cardiac insulin signaling. As a result, glucose was diverted away from cardiomyocytes to tumor cells, promoting cardiac atrophy and heart failure. Interestingly, cardiomyocyte responsiveness to insulin remained intact, suggesting that lack of insulin availability, rather than insulin resistance, contributes to terminal cancer-induced cardiac wasting and failure. Adverse effects of insulin depletion are mediated in part by disturbance of protein kinase B (Akt) signaling, a major pathway in cardiac growth, metabolism, and function. As insulin did not stimulate glucose uptake or proliferation of either B16F10 or C26 tumor cells, systemic insulin supplementation may be a beneficial treatment addition to protect cardiac (and other organ) function in advanced cancer. This idea is supported by findings in the present study, in which low-dose systemic insulin supplementation in both mouse tumor models attenuated cardiac atrophy and dysfunction and lowered tumor growth.

\section{Results}

High tumor burden is associated with low serum insulin in patients with advanced malignant tumors. Unstimulated blood insulin levels were measured in patients with advanced cancer and age- and gender-matched healthy controls (patient characteristics are provided in Supplemental Table 1; supplemental material available online with this article; https://doi.org/10.1172/jci.insight.93098DS1). Blood insulin levels were markedly lower in cachectic cancer patients compared with healthy controls (Figure 1A).

Mice with severe melanoma or colon cancer exhibit progressive cachexia and mortality. Mice implanted i.p. with B16F10-C57BL/6 (B16F10-TM) or C26-BALB/c (C26-TM) tumor cells developed severe abdominal tumor expansion and exhibited significant cancer-related mortality within 14 to 21 days (Figure 1B). Average body weights in advanced disease were markedly lower compared with respective healthy controls (Table 1), despite food intake being normal in B16F10-TM mice and slightly less in C26-TM mice (Supplemental Figure 1), confirming cancer-induced cachexia.

B16F10-TM and C26-TM mice display progressive cardiac dysfunction and atrophy. Serial echocardiography revealed reduced left ventricular (LV) fractional shortening, cardiac output, LV stroke volume, and diastolic endocardial volume in B16F10-TM and C26-TM mice with advanced cancer compared with controls (Table 1). Heart rate was decreased in B16F10-TM and C26-TM mice (Table 1). In addition, cardiac atrophy with lower relative heart weight was apparent compared with strain-matched controls (Figure 1C and Table 1). Histology revealed a smaller cardiomyocyte cross-sectional area in both models (Table 1), associated with modestly reduced $\alpha$ myosin heavy chain (Myho) and increased $\beta$ myosin heavy chain (Myh7) expression (Figure 1, D and E); however, no significant changes in atrial natriuretic peptide ( $A N P$, data not shown) were found in either model. There was no evidence of cardiac inflammation (CD45 immunostaining), fibrosis (H\&E staining), or apoptosis [cleaved caspase-3; cleaved poly(ADP-ribose) polymerase 1 (PARP1) protein] in either tumor model (Supplemental Figure 1). However, markers for proteasomal degradation (Atrogin1 and muscle-specific ring finger protein $1[M u R F 1]$ ) and autophagy (cathepsin $\mathrm{L}$ [CatL] and microtubule-associated protein 1 light chain $3 \beta[L C 3 b]$ ) were elevated (Figure 1, F-I). Moreover, enhanced conversion of $\mathrm{LC} 3 \mathrm{bI}$ into lipidated LC3bII, assessed as increased ratio of LC3bII to LC3bI, was present in both tumor models (Figure 1, J-M), indicating enhanced activation of autophagy.

B16F10 and C26 tumor growth affects cardiac energy substrate uptake. Serial whole body ${ }^{18} \mathrm{~F}$-fluorodeoxyglucose $\left({ }^{18} \mathrm{~F}-\mathrm{FDG}\right)$ positron emission tomography (PET) scans identified a progressive increase in metabolically active tumor volume in the peritoneal cavities of B16F10-TM and C26-TM mice with disease progression (Figure 2, A-D, and Supplemental Figure 2). Tumor growth by ${ }^{18}$ F-FDG-PET scan was also confirmed by bioimaging using in vivo optical imaging system (IVIS, Supplemental Figure 2). In B16F10-TM mice, cardiac glucose uptake $\left({ }^{18} \mathrm{~F}-\mathrm{FDG}\right.$ accumulation) gradually decreased during cancer progression (Figure 2 , A and E). By contrast, cardiac glucose uptake was increased in C26-TM mice with advanced disease (Figure 2, $\mathrm{C}$ and F). Cardiac triglyceride levels were significantly lower compared with strain-matched controls in both models (Figure 2, G and H). Glucose transporter (GLUT) expression in LV tissue was different in the 
A

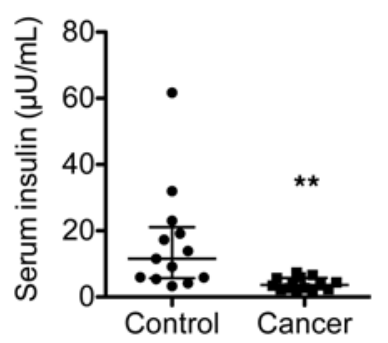

D $\quad$ Control

- B16F10-TM

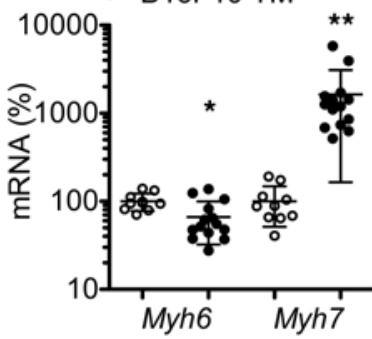

G Control

- C26-TM

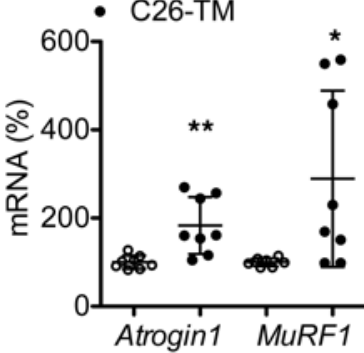

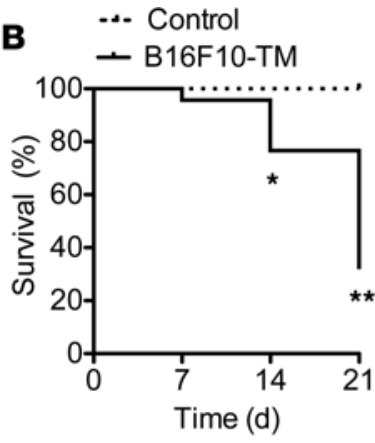

E

E Control

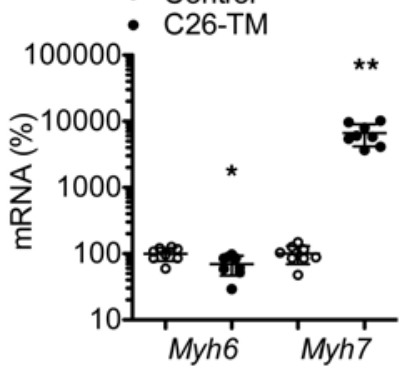

H
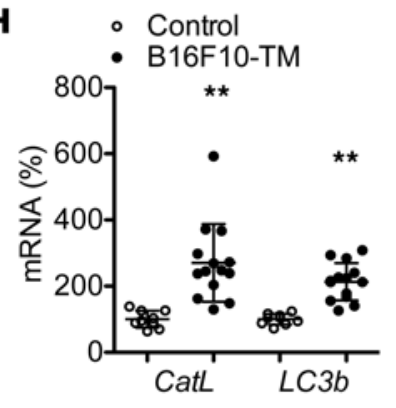

$\mathbf{L}$

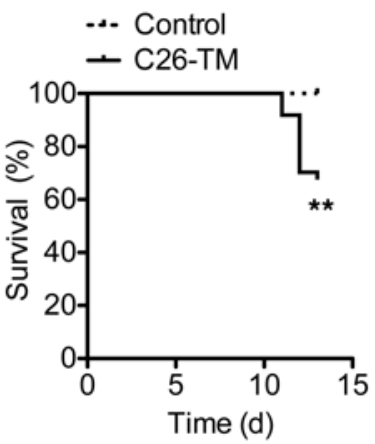

F

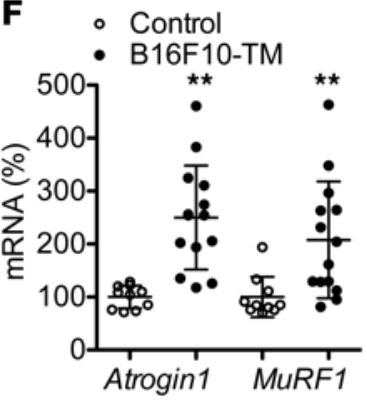

I - Control

1500 - C26-TM

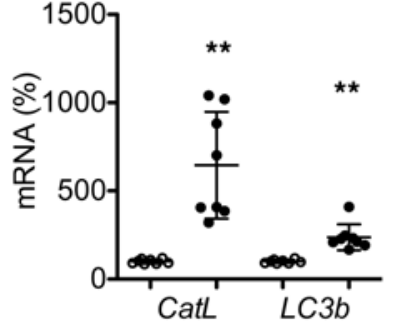

C
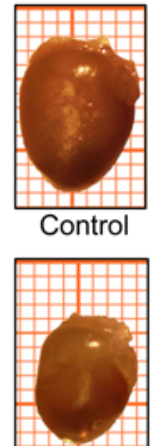

B16F10-TM

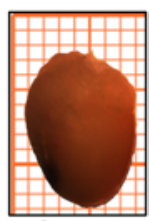

Control

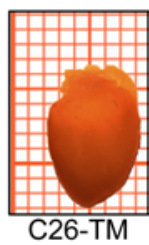

J

K

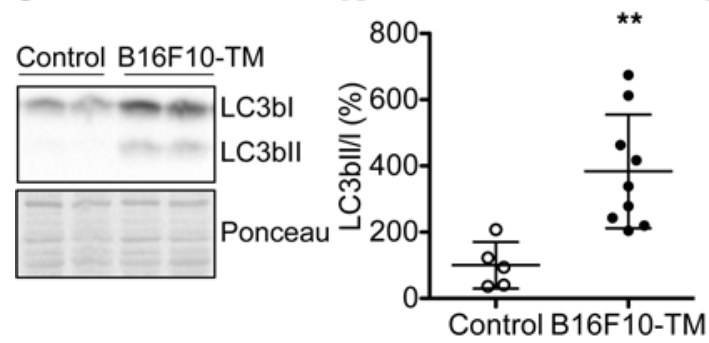

Control C26-TM

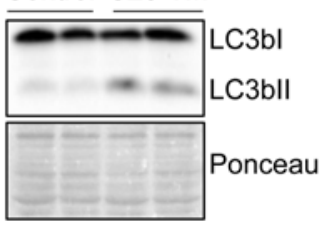

M

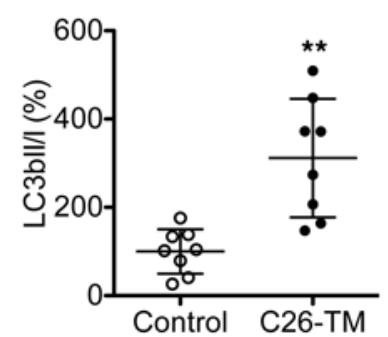

Figure 1. Analyses of blood insulin levels in cancer patients and cardiac phenotype in mice with B16F10 melanoma or C26 carcinoma. (A) Serum insulin levels in cachectic cancer patients $(n=14)$ compared with healthy controls $(n=13)$. (B) Kaplan-Meyer survival curves for C57BL/6 mice with B16F10 melanoma tumors (B16F10-TM) $(n=46)$ and BALB/c mice with colon 26 carcinoma (C26-TM) $(n=37)$ versus corresponding healthy controls ( $n$ $=17$ each). (C) Representative explanted hearts (each square $=1 \mathrm{~mm}^{2}$ ) of B16F10-TM and C26-TM mice and controls. (D-I) Quantitative mRNA levels ( $q R T-P C R)$ of ( $\mathbf{D}$ and $\mathbf{E}$ ) myosin heavy chain isoforms $\alpha$ (Myh6) and $\beta$ (Myh7), ( $\mathbf{F}$ and $\mathbf{G}$ ) atrophy marker Atrogin1 and muscle-specific ring finger protein $1($ MuRF1), and ( $\mathbf{H}$ and $\mathbf{I})$ autophagy markers cathepsin $\mathrm{L}($ Cat $L)$ and microtubule-associated protein 1 light chain $3 \beta$ (LC3b) in B16F10-TM mice ( $n=$ 14 vs. control $n=10$ ) or C26-TM mice and corresponding controls ( $n=8$ each). (J and $\mathbf{L}$ ) Western blots depicting protein levels and Ponceau $S$ staining as loading control for LC3bl and LC3bll. (K and M) LC3blı/LC3bl ratio in left ventricular (LV) tissue from B16F10-TM mice $(n=9)$ and controls $(n=5)$ and C26-TM mice and controls ( $n=8$ each). B16F10-TM and C26-TM mice in C-M were analyzed at an advanced disease stage (14-21 days after tumor inoculation). Healthy control mice were injected with PBS as vehicle. Data are depicted as median with interquartile range (A) or mean \pm SD (D-I, K, and $\mathbf{M}$ ); ${ }^{*} P<0.05,{ }^{* *} P<0.01$ vs. respective healthy controls, using 2-tailed Student's unpaired $t$ tests with or without Welch's correction as required or Mann-Whitney test as required (A, D-I, K, and $\mathbf{M}$ ) or log-rank (Mantel-Cox) test (B). 
Table 1. Morphometry and cardiac function in B16F10-TM and C26-TM mice

\begin{tabular}{|c|c|c|c|c|c|c|}
\hline & Control & B16F10-TM & No. of mice & Control & C26-TM & No. of mice \\
\hline $\mathrm{BW}(\mathrm{g})$ & $28 \pm 1$ & $24 \pm 2^{A}$ & $n=15-26$ & $25 \pm 1$ & $19 \pm 1^{A}$ & $n=16-24$ \\
\hline $\mathrm{HW}$ (mg) & $124 \pm 20$ & $88 \pm 8^{A}$ & $n=15-26$ & $99 \pm 6$ & $73 \pm 8^{A}$ & $n=16-24$ \\
\hline HW/TL (mg/mm) & $7.5 \pm 1.2$ & $5.3 \pm 0.5^{A}$ & $n=15-26$ & $5.9 \pm 0.2$ & $4.5 \pm 0.4^{A}$ & $n=12-16$ \\
\hline CSA (\%) & $100 \pm 15.4$ & $74.8 \pm 2.8^{\mathrm{B}}$ & $n=3-5$ & $100 \pm 9.2$ & $84.4 \pm 1.9^{B}$ & $n=3-5$ \\
\hline FS (\%) & $35 \pm 3$ & $27 \pm 6^{A}$ & $n=16-20$ & $35 \pm 3$ & $26 \pm 5^{A}$ & $n=9-10$ \\
\hline LVEDD (mm) & $3.7 \pm 0.2$ & $3.4 \pm 0.3^{A}$ & $n=16-20$ & $3.3 \pm 0.2$ & $2.8 \pm 0.3^{A}$ & $n=9-10$ \\
\hline LVESD (mm) & $2.4 \pm 0.2$ & $2.4 \pm 0.3$ & $n=16-20$ & $2.2 \pm 0.2$ & $2.1 \pm 0.2$ & $n=9-10$ \\
\hline HR (bpm) & $549 \pm 21$ & $476 \pm 100^{A}$ & $n=16-20$ & $476 \pm 85$ & $346 \pm 95^{A}$ & $n=9-10$ \\
\hline $\mathrm{CO}$ (ml/min) & $15.59 \pm 2.63$ & $8.52 \pm 3.07^{A}$ & $n=16-20$ & $17.59 \pm 1.49$ & $5.29 \pm 2.7^{A}$ & $n=9-10$ \\
\hline $\mathrm{ESV}(\mu \mathrm{l})$ & $28.51 \pm 3.53$ & $18.06 \pm 4.71^{A}$ & $n=16-20$ & $34.28 \pm 1.93$ & $14.64 \pm 5.1^{A}$ & $n=9-10$ \\
\hline EVd $(\mu l)$ & $38.45 \pm 4.78$ & $28.57 \pm 5.96^{A}$ & $n=16-20$ & $39.95 \pm 1.64$ & $20.48 \pm 7.14^{A}$ & $n=9-10$ \\
\hline EVs $(\mu \mathrm{l})$ & $9.94 \pm 2.85$ & $10.45 \pm 6.14$ & $n=16-20$ & $5.67 \pm 0.98$ & $3.84 \pm 3.13$ & $n=9-10$ \\
\hline
\end{tabular}

BW, body weight; HW, heart weight; TL, tibia length; CSA, cross-sectional area; FS, fractional shortening; LVEDD, left ventricular end-diastolic diameter; LVESD, left ventricular end-systolic diameter; HR, heart rate; CO, cardiac output; ESV, endocardial stroke volume; EVd, endocardial diastolic volume; EVs, endocardial systolic volume. Healthy controls are strain-matched to tumor bearing mice (C57BL6 for B16F10-TM and BALB/c for C26-TM, respectively) and severely diseased B16F10-TM and C26-TM mice. Data represent mean \pm SD. ${ }^{A} P<0.01,{ }^{B} P<0.05$ vs. respective healthy control using 2 -tailed Student's paired or unpaired $t$ tests.

two tumor models, with a significant decrease of insulin-dependent GLUT4 in B16F10-TM mice and a significant increase in insulin-independent GLUT1 and a slight increase in GLUT4 in C26-TM mice (Figure 2, I-L). Both tumor models exhibited increased fatty acyl translocase CD36 expression (Figure 2, I-L).

Advanced tumor burden causes low plasma insulin and glucose levels in B16F10-TM and C26-TM mice. Fed-state blood glucose levels demonstrated a progressive decline in tumor-bearing mice (Supplemental Figure 3), despite maintained nutritional intake. Oral glucose stimulation revealed moderately lower blood glucose concentration and markedly lower plasma insulin levels in both B16F10-TM and C26-TM mice compared with controls at 15 minutes after glucose administration (Figure 3, A-D). Unstimulated plasma insulin levels in both models were also reduced compared with respective controls $(-72 \%$ in B16F10-TM [ $n=7], P<0.01$, and $-66 \%$ in C26-TM $[n=6], P<0.01)$. Both the B16F10 tumor cell line as well as the C26 cell line secreted substantial amounts of insulin-degrading enzyme (IDE; Supplemental Figure 3). Reduced systemic insulin was associated with an upregulation of insulin receptor substrate-2 mRNA (Irs2: 13-fold for B16F10-TM [ $n=$ 12], $P<0.01$, and 6-fold for C26-TM [ $n=5], P<0.05)$ compared with healthy controls $(n=5-8)$. Activation of Akt, a downstream mediator of insulin signaling that has been implicated in abnormal glucose uptake and muscle atrophy (12), was markedly reduced in both tumor models (Supplemental Figure 3).

Acute insulin administration increases cardiac glucose uptake and reduces tumor glucose uptake. To evaluate insulin responsiveness, we injected insulin (6 mU/g, i.p.) prior to ${ }^{18} \mathrm{~F}-\mathrm{FDG}$ PET in mice with advanced cancer. Insulin administration resulted in recovery of myocardial ${ }^{18} \mathrm{~F}-\mathrm{FDG}$ uptake in B16F10-TM mice toward levels observed in healthy controls and further increased cardiac glucose uptake in C26-TM mice (Figure 3, E, F, H, and I), indicating intact cardiac insulin response in both models. Acute insulin injections were paralleled by a significant decrease in tumor ${ }^{18} \mathrm{~F}-\mathrm{FDG}$ uptake in B16F10-TM mice and a nonsignificant decrease toward lower ${ }^{18} \mathrm{~F}-\mathrm{FDG}$ uptake in C26-TM mice (Figure 3, G and J).

Insulin response is intact in cardiomyocytes from tumor mice. Insulin sensitivity was validated in vitro in isolated cardiomyocytes from B16F10-TM and C26-TM mice. In both cases, supplementation of culture medium with insulin $(50 \mathrm{nM})$ evoked a profound increase in ${ }^{18} \mathrm{~F}$-FDG uptake (Figure 3, K and L). Of note, however, ${ }^{18} \mathrm{~F}-\mathrm{FDG}$ uptake in isolated cardiomyocytes was lower in C26-TM mice compared with cardiomyocytes from healthy respective controls (Figure 3L). This finding was in contrast with the in vivo observation, suggesting that increased glucose uptake in C26-TM hearts may derive from noncardiomyocytes cells. Indeed, supernatant from C26 cell cultures markedly increased ${ }^{18} \mathrm{~F}$-FDG uptake in endothelial cells (Supplemental Figure 3).

Chronic insulin supplementation attenuates cardiac atrophy and improves cardiac glucose uptake in both tumor models. To test whether insulin depletion was responsible for adverse effects of advanced cancer on the heart, B16F10-TM and C26-TM mice were supplemented with insulin by subcutaneous implantation of insulin-releasing pellets that release low levels of insulin early after tumor inoculation (at day 7 and day 4, respectively). 
A

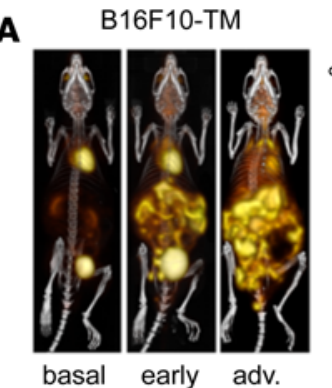

E
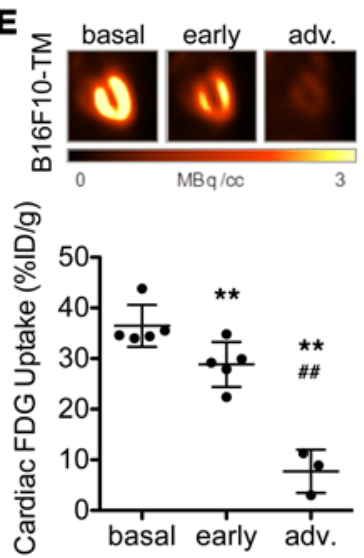

\section{H}

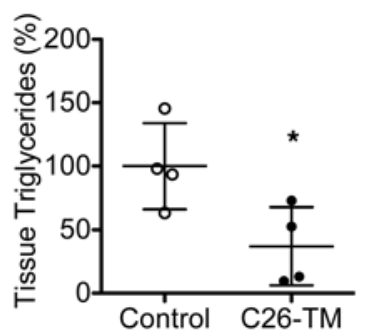

K

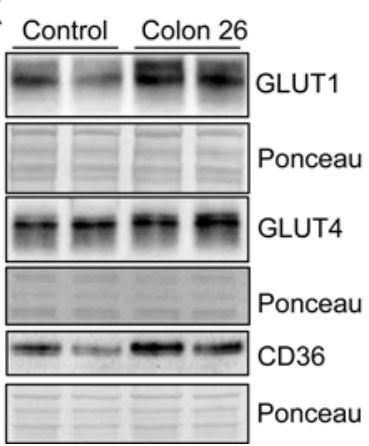

B

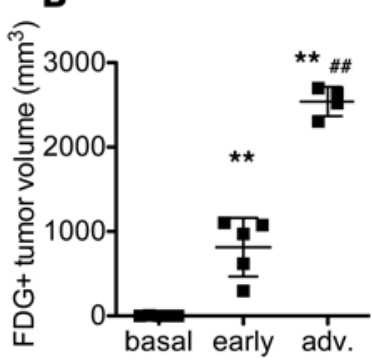

$\mathbf{F}$

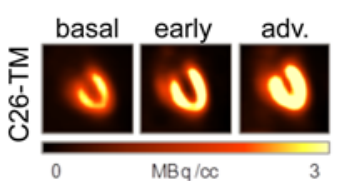

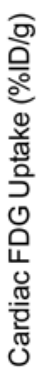$$
\text { I }
$$

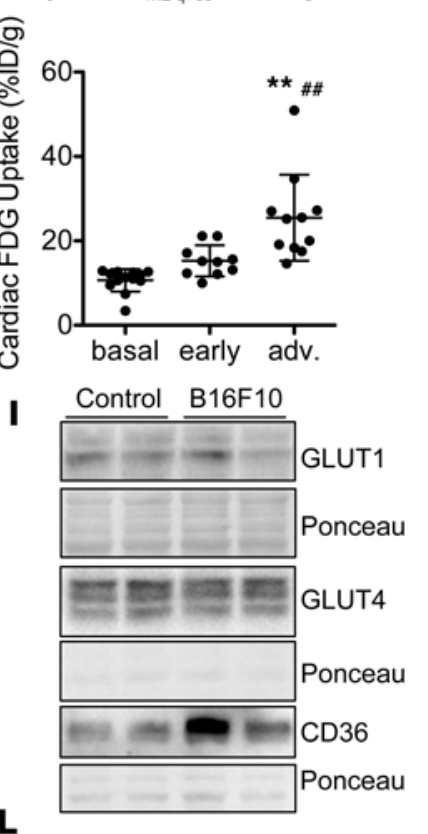

$\mathbf{L}$

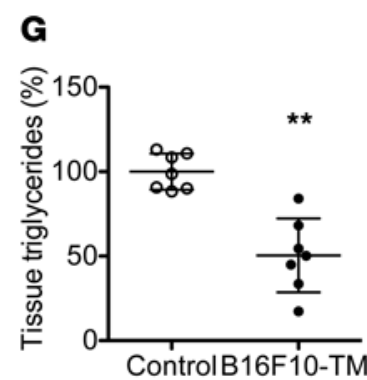

J

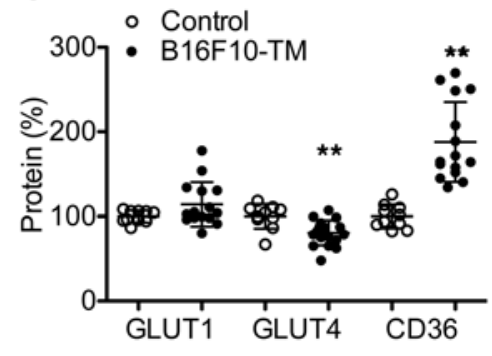

Figure 2. Effect of progressive tumor growth on myocardial glucose uptake and metabolism. (A and C) Serial 3D maximum intensity projection ${ }^{18} \mathrm{~F}$-fluorodeoxyglucose PET-computed tomography $\left({ }^{18} \mathrm{~F}\right.$-FDG PET-CT) images of representative B16F10-TM or C26-TM mice, (B and D) ${ }^{18} \mathrm{~F}-\mathrm{FDG}-\mathrm{derived}$ metabolic tumor volume, and ( $\mathbf{E}$ and $\mathbf{F}$ ) serial representative long-axis myocardial ${ }^{18} \mathrm{~F}$-FDG PET images, with graphs summarizing quantitative uptake (percentage injected dose per gram tissue [\%/D/g]) before tumor inoculation (basal) and at early (day 7 after tumor cell inoculation) and at advanced disease stage (adv.; day 11-17) in B16F10-TM $(n=3-5)$ and C26-TM $(n=13)$ mice. ( $\mathbf{G}$ and $\mathbf{H})$ Triglyceride levels in myocardial tissue of B16F10-TM mice and respective controls ( $n=7$ each) or C26-TM mice versus corresponding controls ( $n=4$ each). (I and $\mathbf{K}$ ) Western blots and ( and $\mathbf{L})$ quantitative protein levels normalized to Ponceau S staining of glucose transporters GLUT1 and GLUT4 and fatty acid translocase CD36 in B16F10-TM mice ( $n=15-18$ ) and controls $(n=10)$ and in C26-TM mice and controls $(n=8$ each), all analyzed at advanced disease stage. Healthy control mice were injected with PBS as vehicle. Data are depicted as mean $\pm \mathrm{SD} ;{ }^{*} P<0.05$, ${ }^{* *} P<0.01 \mathrm{vs}$. respective control or basal state, $\# \#<0.01$ vs. early disease stage, using either 2-tailed Student's unpaired $t$ tests with Welch's correction as required $(\mathbf{G}, \mathbf{H}, \mathbf{J}$, and $\mathbf{L}$ ) or 1-way ANOVA followed by Bonferroni post-hoc tests as required (B, D, E, and $\mathbf{F})$. 
A
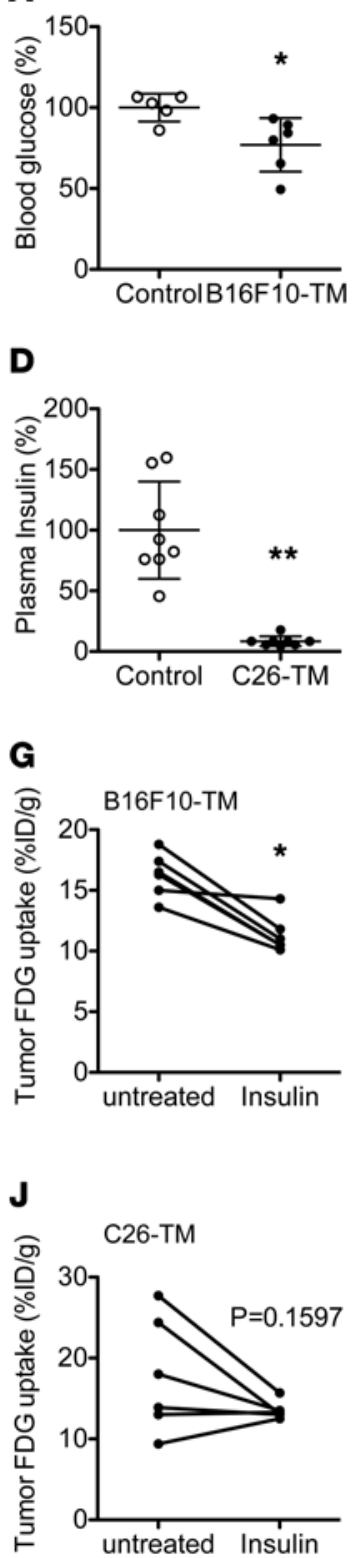

B

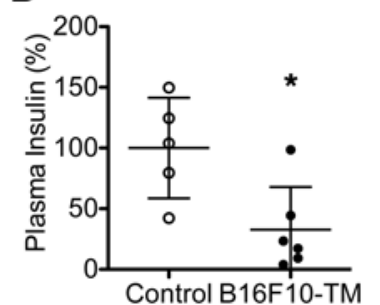

E
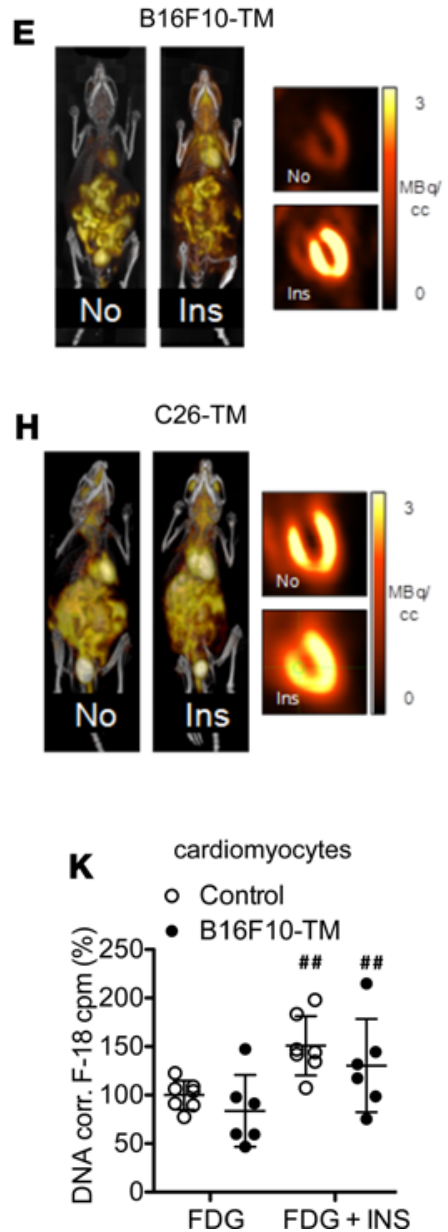

C
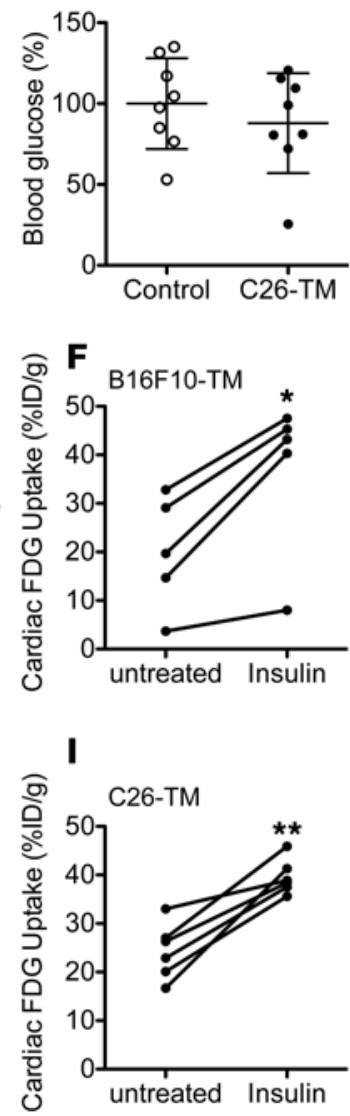

L cardiomyocytes

- Control

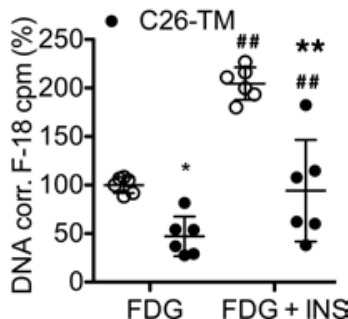

Figure 3. Effect of acute insulin treatment on cardiac and tumor glucose uptake in B16F10-TM and C26-TM mice. (A and C)

Blood glucose concentration after oral glucose load ( $3 \mathrm{mg} / \mathrm{g}$, 15 minutes after loading) and ( $\mathbf{B}$ and $\mathbf{D})$ corresponding plasma insulin concentrations in B16F10-TM mice ( $n=6$ vs. control, $n=5$ ) and C26-TM mice (vs. control, $n=8$ each). (E and $\mathbf{H}$ ) Sample $3 \mathrm{D}$ maximum intensity projection ${ }^{18} \mathrm{~F}$-FDC PET-CT and coronal myocardial images obtained in representative B16F10-TM or C26-TM mice, without (No) and with insulin (Ins) pretreatment $(6 \mathrm{mU} / \mathrm{g} ; 30$ minutes prior to scan) on consecutive days. (F and $\mathbf{I}$ ) Semiquantitative serial assessment of heart and ( $\mathbf{G}$ and $\mathbf{J}$ ) of the tumor ${ }^{18} \mathrm{~F}$-FDG uptake (percentage injected dose per gram of tissue $[\% / D / g]$ ) without and with insulin in B16F10-TM $(n=5)$ and C26-TM $(n=6)$ mice. ( $\mathbf{K}$ and $\mathbf{L}){ }^{18} \mathrm{~F}$-FDG uptake within 60 minutes in isolated cardiomyocytes from B16F10-TM ( $n=6$ vs. control, $n=7$ ) and C26-TM (vs. control, $n=6$ each) mice, with and without insulin (INS, $50 \mathrm{nM}$ ); stimulation was measured in triplicates for each individual and corrected to DNA content. Healthy control mice were injected with PBS as vehicle. Data are depicted as mean $\pm \mathrm{SD} ;{ }^{*} P<0.05,{ }^{* *} P$ $<0.01$ vs. control or untreated mice, ${ }^{\# \#} P<$ 0.01 vs. unstimulated cardiomyocytes, using either 2-tailed Student's unpaired (A-C) or paired (F, G, I, and $\mathbf{J}) t$ tests, Mann-Whitney (D) test, or 2-way ANOVA followed by Bonferroni post-hoc tests as required ( $\mathbf{K}$ and $\mathbf{L}$ ).

Insulin supplementation restored basal blood insulin in B16F10-TM mice (to $99 \% \pm 46 \%, n=8$ vs. $n=6$ controls) and C26-TM mice (to $158 \% \pm 72 \%, n=6$ vs. $n=6$ controls). Cardiac Akt phosphorylation was restored in both models by insulin supplementation (Figure 4, A-D). Exogenous insulin treatment also attenuated cardiac atrophy in both models (Table 2) and reduced the expression of Atrogin 1 and LC3b and the LC3bII/ LC3bI protein ratio (Figure 4, E-L), reflecting attenuated autophagy. Cardiac function, as measured by echocardiography, was significantly improved by insulin supplementation in C26-TM mice (Table 2). In addition, myocardial glucose uptake was stimulated by exogenous insulin treatment, as evidenced by the increase of ${ }^{18} \mathrm{~F}-\mathrm{FDG}$ uptake in B16F10-TM mice and a nonsignificant further increase over untreated in C26-TM mice (Figure 5, A-D), demonstrating intact insulin-sensitive glucose transport in both models.

Insulin supplementation lowers tumor burden in both models, and B16F10 and C26 tumor cells do not respond to insulin stimulation. Chronic insulin supplementation in vivo resulted in a lower tumor burden in both models, measured as ${ }^{18} \mathrm{~F}-\mathrm{FDG}$-avid volume in the peritoneal cavity compared with placebo pellet-treated mice, though ${ }^{18} \mathrm{~F}$-FDG uptake by individual tumors in vivo remained similar (Figure 5, A-D). In line with in vivo data, insulin treatment neither induced glucose uptake nor promoted proliferation in B16F10 or $\mathrm{C} 26$ tumor cells in vitro (Figure 5, E-H). 
Table 2. Chronic insulin supplementation attenuates morphometry changes and cardiac dysfunction in B16F10-TM and C26-TM mice

\begin{tabular}{|c|c|c|c|c|c|c|}
\hline & Placebo B16F10-TM & Insulin B16F10-TM & No. of mice & Placebo C26-TM & Insulin C26-TM & No. of mice \\
\hline $\mathrm{BW}(\mathrm{g})$ & $25 \pm 2$ & $27 \pm 1^{A}$ & $n=7-8$ & $20 \pm 2$ & $20 \pm 1$ & $n=12-13$ \\
\hline $\mathrm{HW}$ (mg) & $87 \pm 11$ & $97 \pm 3^{A}$ & $n=7-8$ & $83 \pm 7$ & $98 \pm 10^{B}$ & $n=12-13$ \\
\hline $\mathrm{HW} / \mathrm{TL}(\mathrm{mg} / \mathrm{mm})$ & $5.1 \pm 0.7$ & $5.7 \pm 0.2^{A}$ & $n=7-8$ & $5.2 \pm 0.4$ & $6.1 \pm 0.6^{\mathrm{B}}$ & $n=12-13$ \\
\hline FS (\%) & $25 \pm 6$ & $28 \pm 3$ & $n=7$ each & $24 \pm 4$ & $34 \pm 3^{B}$ & $n=7$ each \\
\hline
\end{tabular}

BW, body weight; HW, heart weight; TL, tibia length; FS, fractional shortening. All values are from B16F10-TM or C26-TM mice with advanced cancer treated with placebo (palmitic acid) or insulin pellets from 7 days (B16F10-TM) or 4 days (C26-TM) after tumor inoculation. Data were collected at day 14-15 after tumor cell implantation for B16F10-TM mice and at day $11-12$ after tumor cell implantation for C26-TM mice. Data represent mean \pm SD. ${ }^{A} P<0.05,{ }^{B} P<0.01$ vs. respective placebo-treated tumor mice using 2-tailed Student's unpaired $t$ tests.

\section{Discussion}

Impaired insulin signaling has been linked to the development of cardiomyopathy, as evidenced in diabetic animal models and patients $(13,14)$. Here, we report that cachectic cancer patients display markedly reduced serum insulin levels. Similarly, two mouse models with progressive cancer-induced cachexia, caused by either B16F10 melanoma or C26 carcinoma, exhibit markedly reduced systemic insulin levels, which appear partially responsible for cardiac metabolic, morphologic, and functional impairment. It is known that insulin signaling not only controls glucose uptake, but is also crucial for maintaining the balance between anabolic and catabolic pathways in the heart (12). Here, we observed that the reduction of systemic insulin in cancer is in part responsible for impaired cardiac glucose uptake, upregulated catabolic pathways, atrophy, and dysfunction of the heart, as evidenced by the attenuation of these changes by exogenous insulin supplementation in both B16F10-TM and C26-TM mice. Moreover, low serum insulin levels seem to promote tumor growth, since insulin supplementation lowers tumor burden.

Insulin depletion in progressive cancer itself is in part caused by the massive absorption of glucose by the tumor mass, which thereby lowers circulating glucose levels and suppresses insulin secretion, as pancreatic $\beta$ cells depend on active glucose sensing via GLUT2 transport and $\mathrm{K}_{\mathrm{ATP}}$ channel activation (15). Furthermore, it seems that insulin is also actively removed from the system by B16F10 melanoma and C26 carcinoma, as these tumors release high levels of IDE, which efficiently degrades insulin. As a direct indicator of missing insulin signaling, Irs2 expression was markedly increased in hearts of both tumor models. Interestingly, cardiomyocytes in both tumor models respond well to insulin stimulation in vivo and ex vivo, indicating that low systemic insulin and not cardiomyocyte insulin resistance is primarily responsible for impaired cardiac insulin signaling in B16F10-TM and C26-TM mice. Moreover, systemic insulin supplementation in mice with advanced tumor burden even seems to attenuate tumor growth by redirecting glucose to other organs, such as the heart and skeletal muscle. Despite a reduction in FDG-positive tumor burden, the uptake of glucose by individual tumor masses remained consistent, suggesting that tumor growth is arrested at the start of insulin supplementation (i.e., 4-7 days after cell injection), but metabolic activity was maintained in the existing tumor. This observation may also reflect the late analysis time point (50-60 minutes after 18F-FDG administration), as time activity curves indicate earlier saturation of 18F-FDG uptake (i.e., within 30 minutes) (16). Notably, in both tumor cell types (B16F10 and C26), glucose uptake was insulin independent and addition of insulin did not stimulate glucose uptake or cell proliferation.

Insulin supplementation increases glucose uptake by the heart in vivo, and addition of insulin enhances glucose uptake by isolated cardiomyocytes ex vivo in both models. However, in vivo PET scans showed that unstimulated cardiac glucose uptake is progressively reduced only in B16F10-TM mice, whereas it was increased in C26-TM mice. Differences in sympathetic tone, loading conditions, levels of substrates other than glucose, tracer availability in blood, or uptake by noncardiomyocyte cells may all contribute to the surprising increase of cardiac glucose uptake in C26-TM mice (17-20). However, isolated cardiomyocytes from C26-TM mice display lower overall glucose uptake ex vivo, i.e., at baseline without stimulation as well as after addition of insulin, suggesting that advanced cancer does impair glucose uptake in cardiomyocytes of C26-TM mice. This observation suggests that systemic effects of the C26 tumor enhance glucose uptake by noncardiomyocytes in hearts of C26-TM mice. Indeed, supernatant from C26 tumor cells strongly induces glucose uptake in endothelial cells, supporting the idea that increased cardiac glucose uptake in C26-TM mice may indeed derive from noncardiomyocytes. The assumption that insulin signaling is defec- 

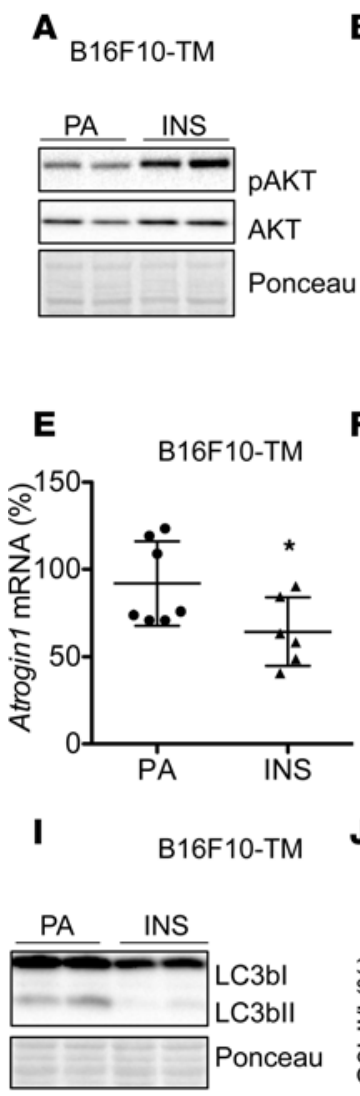

B

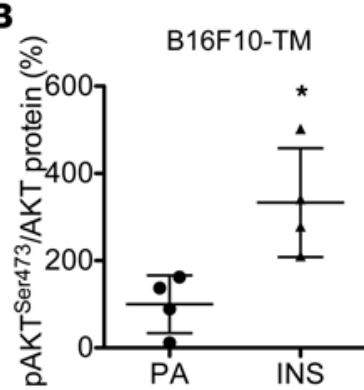

F

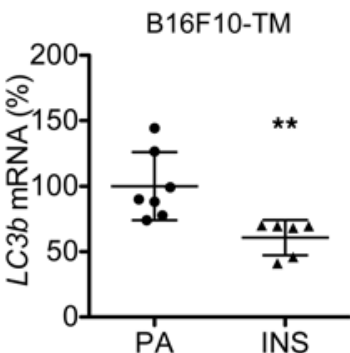

J

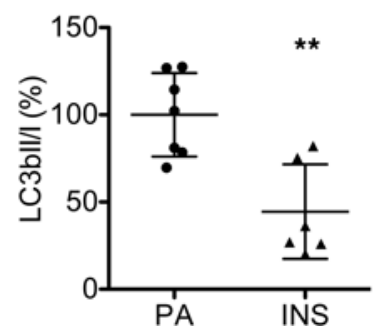

C
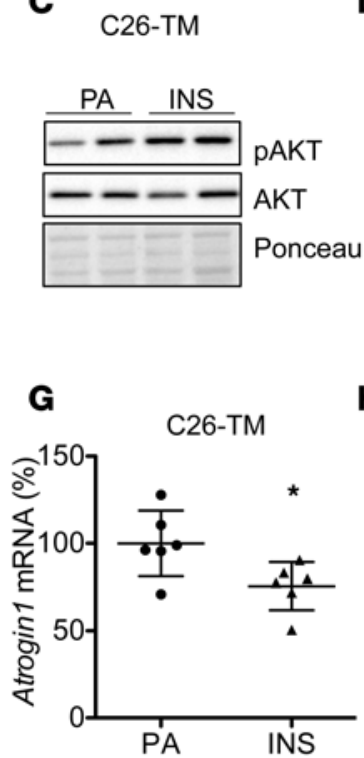

$\mathbf{K}$

C26-TM

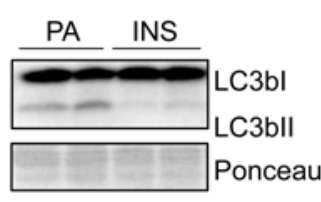

D

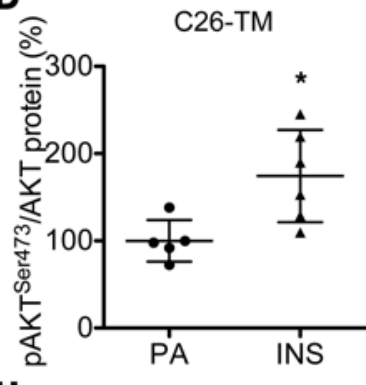

H

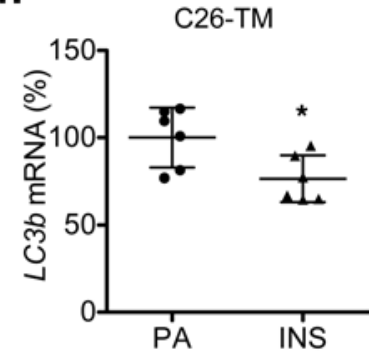

$\mathbf{L}$

C26-TM

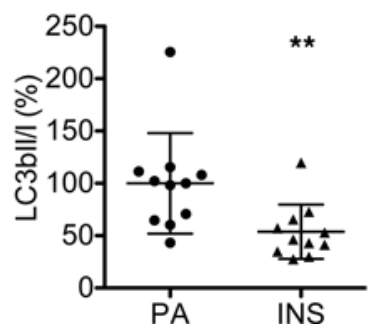

Figure 4. Chronic insulin supplementation attenuates atrophy and autophagy in advanced tumor-bearing mice. (A and C) Western blots showing total Akt and phosphorylation at Ser473 (pAkt) and Ponceau S as loading control in LVs of B16F10-TM and C26-TM mice with advanced cancer treated with insulin-releasing pellets (INS, $0.2 \mathrm{U} / 24$ hours) or carrier substance palmitic acid (PA). (B and D) Ratio of pAkt ${ }^{5473} / A k t$ in B16F10-TM mice with advanced disease treated with PA or INS ( $n=4$ each) or C26-TM mice with advanced disease treated with PA $(n=5)$ or INS $(n=6)$. (E and G) mRNA of Atrogin1 and ( $\mathbf{F}$ and $\mathbf{H}$ ) LC3b in B16F10-TM or C26-TM mice ( $n=6-7$ per group). (I and $\mathbf{K}$ ) Western blots depicting protein levels and Ponceau $S$ staining as loading control for LC3bl and LC3bll. (J and L) The LC3blI/LC3bl ratio in LV tissue from B16F10-TM mice treated with PA $(n=7)$ or INS $(n=6)$ and C26-TM mice treated with PA or INS ( $n=11$ each). Data are depicted as mean \pm SD; ${ }^{A} P<0.05,{ }^{B} P<0.01$ vs. PA-treated B16F10-TM or C26-TM mice using 2-tailed Student's unpaired $t$ tests with (L) or without (B, D-H, and J) Welch's correction

tive in cardiomyocytes from C26-TM mice is further supported by the finding that insulin supplementation markedly attenuates cardiac wasting and improves cardiac function in this tumor model. Taken together, these observations suggest that, although different tumor types may similarly target the insulin signaling pathway in the heart, they may exert different direct effects on individual cardiac cell types.

Our data show that the cardiac impairment caused by insulin depletion in tumor disease differs substantially from insulin resistance in type 2 diabetic cardiomyopathy (21). Tumor-bearing mice do not exhibit the hyperglycemia, hyperinsulinemia, or hyperlipidemia commonly associated with type 2 diabetic models. Additionally, the downstream insulin signaling molecular Irs 2, which is downregulated in diabetic cardiomyopathy (22), is markedly upregulated in both tumor models. Diabetic cardiomyopathy is associated with inflammation, fibrosis, and apoptosis, but no such evidence is present in hearts of the two tumor mouse models studied here. Contractile dysfunction in B16F10-TM and C26-TM mice is largely systolic, i.e., reduced fractional shortening, whereas diabetic cardiomyopathy manifests as diastolic dysfunction and impaired ventricular filling due to interstitial collagen accumulation (23). Finally, in diabetic cardiomyopathy, insulin signaling is impaired at the insulin receptor level, a feature not observed in B16F10-TM and C26-TM mice, as evidenced by intact insulin responsiveness, whereby acute and chronic insulin supplementation evoke a normal response of enhanced glucose uptake in vivo and ex vivo. 
A

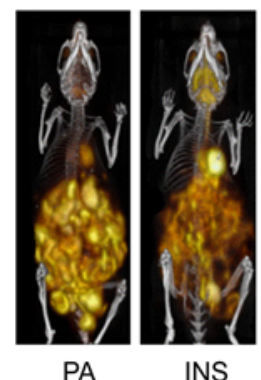

C26-TM

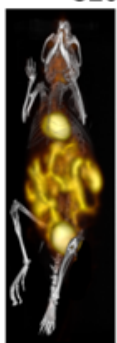

PA

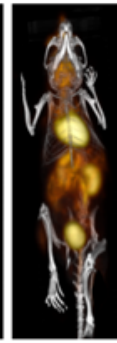

INS

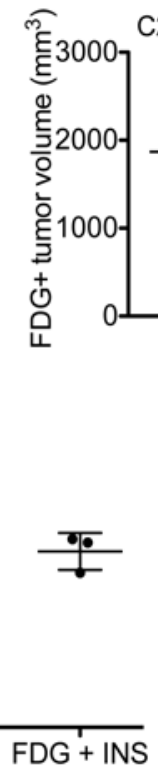

G
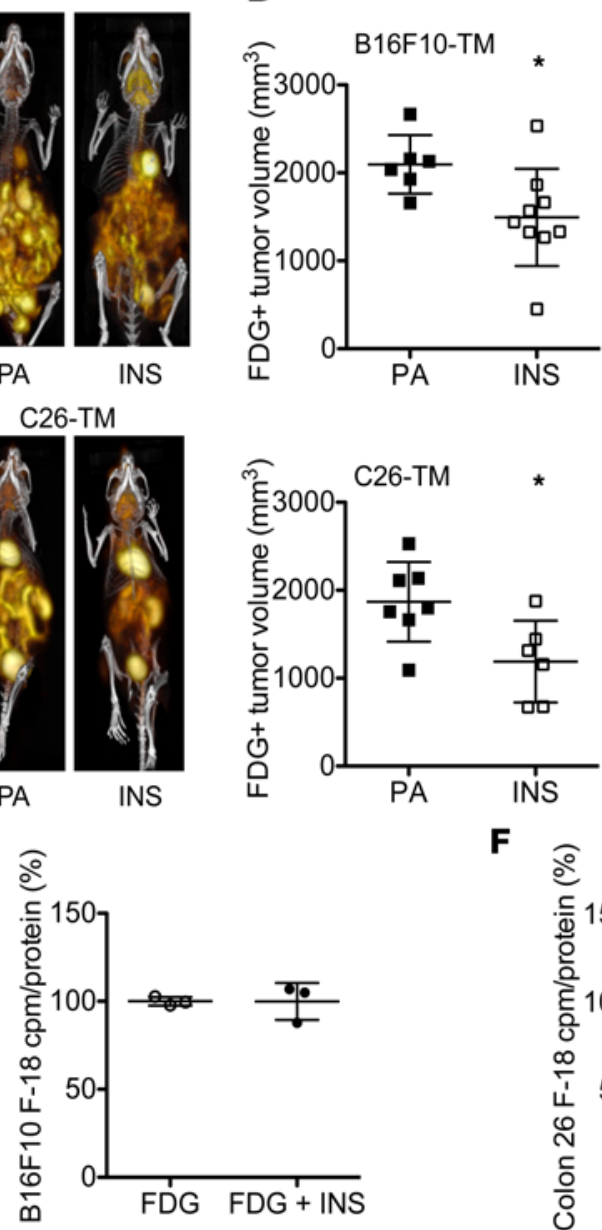

C
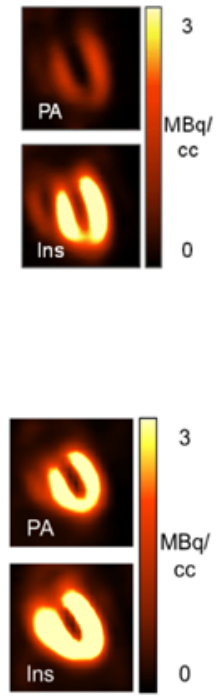

PA INS
D
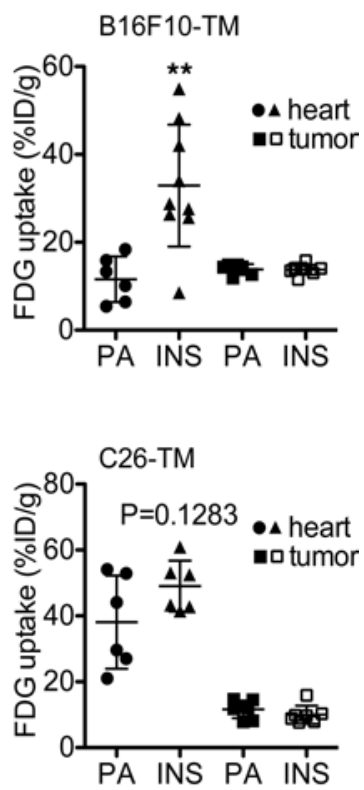

Figure 5. Chronic insulin supplementation attenuates metabolic impairment and metabolically active tumor volume. (A) $3 \mathrm{D}$ maximum intensity projection ${ }^{18} \mathrm{~F}-\mathrm{FDC}$ PET-CT images and (B) derived metabolically active ${ }^{18} \mathrm{~F}$-FDG-avid tumor volume as well as (C) representative coronal slice cardiac ${ }^{18} \mathrm{~F}$-FDC PET images and (D) semiquantitative ${ }^{18} \mathrm{~F}$-FDG uptake (percentage injected dose per gram tissue [\%।D/g]) in hearts and tumors in B16F10TM and C26-TM mice receiving palmitic acid (PA, $n=6-7$ ) or insulin supplementation (INS, $n=6-9$ ). (E and F) ${ }^{18} \mathrm{~F}-\mathrm{FDC}$ uptake of B16F10 and colon 26 cells in in vitro culture without and with insulin stimulation (50 nM) for 1 hour, ( $n=3$ culture dishes per cell line). ( $\mathbf{G}$ and $\mathbf{H}$ ) BrdU incorporation assay combining results from 3 independent experiments with B16F10 and colon 26 cells with and without insulin stimulation (50 nM) for 24 hours. Nonstimulated control cell BrdU incorporation was set to $100 \%$ for each experiment. Data are depicted as mean $\pm \mathrm{SD} ;{ }^{\mathrm{A} P}<0.05,{ }^{\mathrm{B} P}<0.01$ vs. $\mathrm{PA}$ or respective control using either 2-tailed Student's unpaired $t$ tests with (D) or without (B) Welch's correction or 1-sample $t$ test $(\mathbf{H})$.

Impaired myocardial glucose uptake may be counteracted by high metabolic flexibility, whereby cardiomyocytes can metabolize substrates, including fatty acids (24). However, as studied here, in advanced tumor disease, circulating fatty acids are depleted (6), and this feature seems to be present in our tumor models as well, as we found markedly decreased cardiac triglyceride levels, despite increased CD36 expression. Indeed, hypoinsulinemia can impair lipogenesis, as fat storage in adipose tissue relies on GLUT4 for glycolysis and acetyl-CoA production (25). This impairment can therefore impel lipolysis, loss of fat mass, and liberation of nonesterified fatty acids and interfere with cardiac metabolic flexibility. In the present study, recovered myocardial glucose transport by insulin supplementation suggests that insulin depletion is the major impediment to cardiac metabolic flexibility in this model.

Previous studies indicated that insulin signaling not only regulates cardiac glucose uptake, but also suppresses catabolic pathways, i.e., the ubiquitin proteasome system (Atrogin1) and autophagy (LC3b) via Akt (12). Indeed, we observed increased expression of Atrogin1 and LC3b in hearts from B16F10-TM and C26TM mice. This upregulation was associated with reduced activation of Akt, a signaling molecule known 
to suppress protein degradation by both systems. Insulin supplementation restored cardiac Akt activation, lowered expression of Atrogin 1 and LC3b, and decreased the LC3bII/LC3bI ratio, indicating that restored insulin signaling can attenuate tumor-induced catabolism of the heart. Moreover, insulin replacement normalizes glucose uptake and improves cardiac function, further supporting its positive effect on the heart. Importantly, insulin supplementation lowers the tumor burden in both cancer models, suggesting that this treatment, possibly by limiting substrate availability for tumor cells, not only prevents adverse effects of tumor disease on the heart, but also impedes tumor growth.

Our experimental data align with clinical observations of depleted serum insulin levels in patients with advanced cancer-induced cachexia, which may in part underlie some tumor-induced pathologies, such as heart failure and cardiac wasting. Indeed, lower levels of insulin receptor phosphorylation, reflecting reduced insulin stimulation, were associated with higher mortality among a cohort of colorectal cancer patients (26). In a study of 138 cancer patients of variable origin, supplemental treatment with insulin improved carbohydrate metabolism and enhanced 1-year survival (27), though no improvement in exercise capacity or physical activity was noted. Measurements in this trial were limited to skeletal muscle, whereas our current findings suggest that the survival benefit may relate to improved cardiac function. Clinical testing of cardiovascular effects of insulin supplementation in a comparable patient population is warranted. Nevertheless, hormonal modification, particularly the improvement of the insulin/glucagon ratio, has been explored as a potential cancer treatment to impede tumor growth (8), and the present study suggests that such treatments may have the ancillary benefit of restoring cardiac metabolic balance, preventing cardiac atrophy, and improving cardiac function while at the same time limit tumor growth. We suggest that systemic insulin supplementation may be a beneficial treatment addition to protect cardiac (and other organ) function and, therefore, that it may be supportive in cardio-oncologic treatment concepts by limiting cancer-induced cardiac, and possibly also other organ, damage as well as reducing the tumor burden, all features that would improve overall quality of life in patients with cancer.

\section{Methods}

Patient data. Serum samples were collected at the Hannover Medical School. Only cancer patients with a loss of body weight $>5 \%$ within 3-12 months before sample collection were included. The patient collective displayed different cancer types. Control samples were obtained from healthy blood donors. Insulin concentration in serum was determined using a commercial ELISA kit (EZHI-14K Human Insulin, Merck Millipore) following the manufacturer's protocol.

Preparation of tumor cell lines. The murine cancer cell lines B16F10-TM (C57BL/6) and C26 adenocarcinoma $(\mathrm{BALB} / \mathrm{c}$ ) were grown in DMEM culture medium containing $4.5 \mathrm{~g} / 1$ glucose (Biochrom AG) supplemented with 10\% FCS (Biochrom AG) and penicillin/streptomycin (100 U/100 $\mu \mathrm{g}$ per ml, Gibco). The murine endothelial cell line C166 was grown in DMEM culture medium containing $4.5 \mathrm{~g} / 1$ glucose (Biochrom AG) supplemented with 10\% BCS (Biochrom AG) and penicillin/streptomycin (100 U/100 $\mu$ g per $\mathrm{ml}$, Gibco). For injection, tumor cells were grown to confluence and detached from cell culture flasks by treatment with $0.25 \%$ trypsin/EDTA (Gibco). The trypsin reaction was arrested by addition of culture medium, and the cell suspension was transferred to a falcon tube and centrifuged. The cell pellet was washed twice with prewarmed, sterile PBS; then, cells were suspended in PBS and counted. The B16F10 and C166 cell lines were obtained from ATCC. The C26 cell line was purchased from I.A.Z. (Toni Lindl, München, Germany).

Animal experiments. Male C57BL/6N mice (12 \pm 2 weeks of age) were injected with B16F10-TM cells $\left(1 \times 10^{6}\right)$ or PBS as vehicle. Male BALB/c mice $(12 \pm 2$ weeks of age) were injected with C26 adenocarcinoma cells $\left(1 \times 10^{6}\right)$ or vehicle. After cell implantation, mice received continuous analgesia (Novalgin, $1,000 \mathrm{mg} / \mathrm{kg} /$ day in drinking water). Mice were housed in groups of 5 and maintained on a 14-hour/10 hour light/dark cycle with standard laboratory chow and water freely available. Insulin or control palmitic acid pellets (LinShin Canada) were implanted subcutaneously by trocar and stylet (2 pellets per mouse/0.1 $\mathrm{U} / 24$ hours/implant) under light isoflurane anesthesia at 7 days after tumor cell injection for the B16F10 model and at 4 days after tumor cell inoculation for the C26 model. For assessment of food intake, healthy control mice and tumor-bearing mice were individually caged and food consumption was measured by weighing the food pellets in each individual cage over the entire experimental time frame.

The data presented, if not explicitly stated otherwise, result from analyses of mice with advanced cancer (13-21 days for the C57BL/6 B16F10 model and 11-14 days for the BALB/c C26 model when body weight was reduced by $10 \%-15 \%$ of age- and gender-matched healthy controls) compared with strain- 
matched healthy controls. The health condition of animals was assessed based on the guidelines of recognition and distress in experimental animals as proposed by Morton and Griffiths (28). Where possible, data were analyzed by an experimenter blinded to the tumor status and treatment regimen of the animals.

Cardiac function. Contractile function and heart rate were assessed by serial echocardiography using the Vevo 770 system (VisualSonics) in a subset of tumor-bearing mice and corresponding controls at baseline and at advanced disease states as described previously (29). Parasternal short- or long-axis views were recorded in B-mode at the level of the papillary muscle, and still images were used to measure LV end-diastolic diameter (LVEDD) and LV end-systolic diameter (LVESD) and calculate fractional shortening $([L V E D D-$ LVESD]/LVEDD $\times 100)$ and fractional area change ([end-diastolic area - end-systolic area/ end-diastolic area $\times 100$ ). Cardiac output, LV stroke volume, diastolic endocardial volume, and endocardial systolic volume were calculated by Visual Sonics Vevo 770 software version 3.

Serial ${ }^{18}$ F-FDG PET-CT imaging. Dynamic PET scans in nonfasted mice were conducted at baseline (7-14 days before implant) and early (7 days after implant) and advanced cancer time points (11-21 days after implant) under continuous isoflurane anesthesia $\left(1.5 \%\right.$ at $\left.0.61 / \mathrm{min} \mathrm{O}_{2}\right)$ as previously described (30). Briefly, mice were positioned prone on the scanner bed and centered in the PET camera (Inveon DPET, Siemens) field of view. ${ }^{18} \mathrm{~F}-\mathrm{FDG}(7.9 \pm 0.7 \mathrm{MBq})$ was administered via a lateral tail vein catheter as a $0.2-\mathrm{ml}$ bolus in saline. Dynamic listmode data were acquired over 60 minutes. A transmission scan with a ${ }^{57} \mathrm{Co}$ source was completed for the purpose of attenuation correction. Initial scans were conducted without metabolic preparation. Subsequently, to assess the effect of exogenous insulin, B16F10-TM mice and C26TM mice underwent paired ${ }^{18} \mathrm{~F}$-FDG scans at the advanced time point on consecutive days, once without metabolic preparation and once with insulin (Human Insulin Normal 100, Lilly Pharma) pretreatment (6 $\mathrm{mU} / \mathrm{g}, 1 \mathrm{mg} / \mathrm{g}$ glucose, i.p., 30 minutes prior) in a randomized order.

For coregistration purposes and to help in identifying tumor mass, a fast sequence micro-CT was also obtained following each PET scan. Briefly, the scanner bed was transferred to the CT scanner (Inveon CT, Siemens). Acquisition was conducted at $360^{\circ}$ rotation in 180 steps, with 120 -ms exposure time over 3 fields of view, with $20 \%$ overlap ( $49 \mathrm{~mm}$ transaxial, $128 \mathrm{~mm}$ axial). Images were reconstructed to a $512 \times 512 \times$ 512 image matrix (96- $\mu \mathrm{m}$ voxel size). CT acquisitions were conducted without contrast.

Image processing and analysis. Listmode PET data were histogrammed into 32 frames ( 5 frames per 2 seconds, 4 frames per 5 seconds, 3 frames per 10 seconds, 8 frames per 30 seconds, 5 frames per 60 seconds, 4 frames per 300 seconds, and 3 frames per 600 seconds) and reconstructed to a $128 \times 128 \times 159$ image matrix $\left(0.486-\mathrm{mm}^{3}\right.$ voxel size) using an iterative algorithm (ordered subset expectation maximization $3 \mathrm{D}$; maximum a posteriori; 2 OSEM iterations; $18 \mathrm{MAP}$ iterations, $\beta=1$ ). Corrections for decay, scatter, and attenuation were applied according to the manufacturer defaults.

Regional activity distribution was assessed using Siemens Inveon Research Workplace (IRW 2.0) software. Regions of interest (ROI) were defined using set parameters for the myocardium and left atrial blood pool as image-derived input function (16). Tissue ${ }^{18} \mathrm{~F}-\mathrm{FDG}$ uptake at 50-60 minutes after injection was analyzed semiquantitatively, as percentage injected dose per gram $(\% \mathrm{ID} / \mathrm{g})$, and calculated as follows: activity concentration $(\mathrm{Bq} / \mathrm{ml}) /$ injected Dose $(\mathrm{Bq}) \times 100 \%$. Metabolically active tumor was identified semiautomatically, modifying an ROI of the whole body caudal to the diaphragm to omit activity ascribed to the kidneys and bladder. Pixels in this region with activity concentration exceeding background were considered as metabolically active, ${ }^{18} \mathrm{~F}-\mathrm{FDG}-$ avid tumor. In addition to total tumor activity, a single mass of equivalent size within and between subjects was selected as a representative tumor for quantitative analysis. The ${ }^{18} \mathrm{~F}-\mathrm{FDG}-$ based definition of tumor volume was subject to overestimation due to spillover of high activity ${ }^{18} \mathrm{~F}-\mathrm{FDG}$-avid tumor into surrounding pixels, such that uptake (as \% $\mathrm{ID} / \mathrm{g}$ ) should be considered as a complementary measurement.

Bioimaging using in vivo imaging system. B16F10 and C26 cells were transduced with a lentiviral vector SLIEW encoding both enhanced green fluorescent protein, for in vitro analysis, and firefly luciferase, for in vivo bioluminescent imaging (31). Individual clones were isolated, expanded, and analyzed for stable EGFP and luciferase expression. B16F10 or C26 cells $\left(1 \times 10^{6}\right)$ were injected i.p. into C57BL6 or BALB/C mice, respectively. Mice were imaged using an in vivo imaging system (IVIS) Lumina II (Caliper Life Sciences) for 1 minute following i.p. injection of $100 \mu \mathrm{l}$ of $8 \mu \mathrm{g} / \mu \mathrm{l}$ D-Luciferin (AppliChem). Living Image 3.1 software was used to analyze bioluminescence intensity.

Oral glucose stimulation. Healthy control mice and B16F10-TM or C26-TM mice with advanced cancer were fasted for 5 hours. At 15 minutes after oral glucose loading ( $3 \mathrm{mg} / \mathrm{g}$ body weight), mice were sacrificed by cervical dislocation, and blood samples were collected from the right ventricle in vials containing 
EDTA. Blood glucose concentration was measured using a glucose meter (Bayer). Plasma was isolated by centrifugation and stored at $-80^{\circ} \mathrm{C}$. Insulin concentration in plasma was determined using a commercial ELISA kit (EZRMI-13K Rat/Mouse Insulin, Merck Millipore) following the manufacturer's protocol.

Myocardial triglyceride content. Triglyceride content in LV tissue samples was determined using a commercial immunoassay (Abcam, ab65336) following the manufacturer's protocol.

$q R T-P C R$. Total RNA from a subset of adult murine hearts was isolated with Trizol (Invitrogen), and cDNA synthesis was performed as described previously using the Superscript III system (Invitrogen) (29, 32). Real-time PCR using the SYBR green dye method (Maxima Sybr Green qPCR Master Mix, Thermo Scientific, K0253) was performed with the Stratagene MX3005P multiplex QPCR system as described previously $(29,32,33)$. A detailed list of qRT-PCR primers is provided in Supplemental Table 2.

Western blot. Protein expression levels and phosphorylation were determined by Western blotting in a subset of mice, using SDS-PAGE, as previously described (34). For detection of GLUT1 and GLUT4, samples were not boiled before SDS-PAGE. The following antibodies were used: anti-phospho-AKT Ser473 (Cell Signaling, 4060), anti-AKT (Cell Signaling, 9272), anti-caspase-3 (Cell Signaling, 9662), antiCD36 (Abcam, ab80080), anti-GLUT1 (abcam, ab652), anti-GLUT4 (Abcam, ab654), anti-IDE (abcam, ab133561), anti-LC3b (Sigma-Aldrich, 7543), and anti-PARP1 (Cell Signaling, 9542). Quantification of bands was done automatically using the Bio-Rad Chemidoc MP with Image Lab 5.0 software. Total protein staining by Ponceau S was always used to confirm equal loading and for normalization of protein loading.

Histology, morphology, and immunostaining. At the conclusion of experiments, hearts from a subset of mice were frozen for cryosectioning. On LV cryosections, H\&E staining was performed as described previously (35). Inflammatory status was assessed by CD45 (BD Pharmingen, clone 30-F11) immunohistochemistry counterstained with eosin $(29,32)$. Cardiomyocyte cross-sectional area was determined on longitudinal paraffin sections of in situ-fixed hearts of B16F10-TM, C26-TM, and respective control mice after wheat germ agglutinin/ Hoechst staining as previously described $(29,32)$. At least 30 cells per individual heart were measured.

Isolation of adult primary cardiomyocytes and cell culture. Cardiomyocytes were isolated by enzymatic digestion as described previously (36) and plated on laminin-coated tissue culture plates from a subset of mice. Subsequently, cardiomyocytes were cultivated in $1 \times$ MEM culture medium (Gibco) containing $1 \%$ penicillin, $1 \%$ L-glutamine, and $25 \mu \mathrm{M}(-)$-blebbistatin (Cayman Chem, 13013) with $1 \% \mathrm{CO}_{2}$ at $37^{\circ} \mathrm{C}$ in a cell culture incubator.

Cardiomyocyte ${ }^{18} \mathrm{~F}$-FDG uptake. ${ }^{18} \mathrm{~F}$-FDG uptake assays were performed 24 hours after cell isolation according to published methods $(37,38)$. Briefly, ${ }^{18} \mathrm{~F}-\mathrm{FDG}(250 \mathrm{kBq} / \mathrm{ml})$ with or without insulin $(50 \mathrm{nM}$; Human Insulin Normal 100, Lilly Pharma) was added to medium in $100 \mu 1$. Following 60 minutes of incubation at $37^{\circ} \mathrm{C}$, residual medium was removed and cells were washed twice with $1 \mathrm{ml}$ warm glucose-free medium, lysed in $250 \mu \mathrm{DNA}$ isolation buffer (0.05 M TRIS, $0.1 \mathrm{M}$ EDTA, $0.1 \mathrm{M} \mathrm{NaCl}, 1 \% \mathrm{SDS}$ ), and counted in a gamma counter along with a $1 \%$ dilution of the standard injection. Radioactivity was normalized to DNA content in lysates using PCR.

Cell line ${ }^{18} \mathrm{~F}$-FDG uptake. ${ }^{18} \mathrm{~F}$-FDG uptake assays were performed for B16F10 and $\mathrm{C} 26$ cell lines in triplicates. Cells were cultured in FBS-free DMEM culture medium (Biochrom AG) without glucose and L-glutamine for 2 hours prior to experiment. ${ }^{18} \mathrm{~F}$-FDG $(250 \mathrm{kBq} / \mathrm{ml})$ with or without insulin $(50 \mathrm{nM})$ was added to medium at $100 \mu$ l. Following 60 minutes of incubation at $37^{\circ} \mathrm{C}$, residual medium was removed and cells were washed twice with $1 \mathrm{ml}$ warm glucose-free medium and lysed in $100 \mu 1$ RIPA buffer. Protein content was determined by Bradford assay.

For ${ }^{18} \mathrm{~F}$-FDG uptake assays, C166 cells were cultivated with 50\% regular culture medium and 50\% supernatant of the respective tumor cell line for 24 hours. One hour before starting the assay, cells were washed once with PBS and further cultivated with $50 \%$ of the respective tumor cell line supernatant and $50 \%$ DMEM containing $1 \mathrm{~g} / 1$ glucose (Biochrom AG). Cells were cultivated with ${ }^{18} \mathrm{~F}$-FDG for 1 hour and then washed twice with DMEM and lysed in $100 \mu 1$ RIPA buffer. Activity of the lysates was measured, and Bradford assay was used to determine protein content.

BrdU incorporation assay. Cell Proliferation ELISA (Roche, 11647229001) was used in a 96-well format with 2,500 cells/well in FBS-free DMEM with or without $50 \mathrm{nM}$ insulin (Sigma-Aldrich, I0516) according to manufacturer's protocol.

IDE ELISA. Tumor cells (B16F10 and C26) were grown to $70 \%$ confluency on $n=3$ culture dishes for each cell line. Subsequently, cells were incubated in serum-free DMEM for 24 hours, and cell culture supernatants were collected and $4 \times$ concentrated using Amicon Ultra- 4 centrifugal filters (Merck Millipore). 
IDE in concentrated medium was detected using a commercial ELISA kit (mybiosource, MBS 2884110) according to manufacturer's protocol.

Statistics. All data are presented as mean \pm SD. Data were tested for normal distribution by D'Agostino-Pearson omnibus normality test or Shapiro-Wilk normality test and, depending on the result, compared either using Student's 2-tailed unpaired $t$ tests with or without Welch's correction (depending on equal variances) or Mann-Whitney test. Serial imaging data were compared serially within each subject using 2-tailed Student's paired $t$ tests. Multiple group comparisons were made using 1- or 2-way ANOVA with Bonferroni's post-hoc correction. $P<0.05$ was considered as significant.

Study approval. All individuals and patients provided written informed consent for the anonymized storage and further testing of their samples. This study was approved by our institutional review board ( $\mathrm{MHH}$ Ethikkommission) located in Hannover, Lower Saxony, Germany. All animal studies were in accordance with the German animal protection law and with European Communities Council Directive 86/609/EEC and 2010/63/EU for the protection of animals used for experimental purposes. All experiments were approved by the local institutional animal care and research advisory committee and permitted by LAVES (Niedersächsisches Landesamt für Verbraucherschutz und Lebensmittelsicherheit; Oldenburg, Lower Saxony, Germany).

\section{Author contributions}

JTT conceived the study, performed PET-CT and ${ }^{18} \mathrm{~F}-\mathrm{FDG}$ analyses, and drafted the manuscript. SP conceived the study of mouse cancer models, including genetic studies and functional analyses by echocardiography, and performed ex vivo analyses of cardiomyocytes and cell culture experiments. BS conceived the study and performed biochemical analyses. MRH performed histological analyses. CWL performed the IVIS analyses. JPB developed the PET-CT assay and radiopharmaceutical logistics. MS provided biochemical assays. JH collected patients and provided discussion. GS collected patients and handled biobanking. $\mathrm{AH}$ designed the study and provided biochemical assays and clinical discussion. FMB designed the study, provided clinical discussion, and wrote the manuscript. DHK conceived the idea for the project, designed the study, analyzed data, and wrote the manuscript.

\section{Acknowledgments}

The authors thank Martina Kasten, Silvia Gutzke, Sergej Erschow, Mirco Müller, Birgit Brandt, ChunWei Lee, Silvia Eilert, Alexander Kanwischer, and Petra Felsch for excellent technical assistance. JTT is supported by a fellowship from the Canadian Institutes of Health Research. This work was supported by the German Research Association (DFG) (HI-842/10-1, KFO 311, Rebirth II).

Address correspondence to: Denise Hilfiker-Kleiner, Abt. Kardiologie und Angiologie, Medizinische Hochschule Hannover, Carl-Neuberg Strasse 1, 30625 Hannover, Germany. Phone: 49.511.532.2531; E-mail: hilfiker.denise@mh-hannover.de

1. Fearon K, et al. Definition and classification of cancer cachexia: an international consensus. Lancet Oncol. 2011;12(5):489-495.

2. von Haehling S, Anker SD. Cachexia as a major underestimated and unmet medical need: facts and numbers. J Cachexia Sarcopenia Muscle. 2010;1(1):1-5.

3. Murphy KT. The pathogenesis and treatment of cardiac atrophy in cancer cachexia. Am J Physiol Heart Circ Physiol. 2016;310(4):H466-H477.

4. Hill JA, Olson EN. Cardiac plasticity. N Engl J Med. 2008;358(13):1370-1380.

5. Honors MA, Kinzig KP. The role of insulin resistance in the development of muscle wasting during cancer cachexia. $J$ Cachexia Sarcopenia Muscle. 2012;3(1):5-11.

6. Springer J, et al. Prevention of liver cancer cachexia-induced cardiac wasting and heart failure. Eur Heart J. 2014;35(14):932-941.

7. De Blaauw I, Deutz NE, Von Meyenfeldt MF. Metabolic changes of cancer cachexia--second of two parts. Clin Nutr. $1997 ; 16(5): 223-228$.

8. De Blaauw I, Deutz NE, Von Meyenfeldt MF. Metabolic changes in cancer cachexia--first of two parts. Clin Nutr. 1997;16(4):169-176.

9. Evans JM, Donnelly LA, Emslie-Smith AM, Alessi DR, Morris AD. Metformin and reduced risk of cancer in diabetic patients. BMJ. 2005;330(7503):1304-1305.

10. Djiogue S, et al. Insulin resistance and cancer: the role of insulin and IGFs. Endocr Relat Cancer. 2013;20(1):R1-R17.

11. Beluzi M, et al. Pioglitazone treatment increases survival and prevents body weight loss in tumor-bearing animals: possible anti-cachectic effect. PLoS One. 2015;10(3):e0122660.

12. Riehle C, Abel ED. Insulin signaling and heart failure. Circ Res. 2016;118(7):1151-1169.

13. From AM, Scott CG, Chen HH. The development of heart failure in patients with diabetes mellitus and pre-clinical diastolic 
dysfunction a population-based study. J Am Coll Cardiol. 2010;55(4):300-305

14. Radovits T, et al. Comparative investigation of the left ventricular pressure-volume relationship in rat models of type 1 and type 2 diabetes mellitus. Am J Physiol Heart Circ Physiol. 2009;297(1):H125-H133.

15. Hiriart M, Velasco M, Larqué C, Diaz-Garcia CM. Metabolic syndrome and ionic channels in pancreatic beta cells. Vitam Horm. 2014;95:87-114.

16. Thackeray JT, Bankstahl JP, Bengel FM. Impact of image-derived input function and fit time intervals on patlak quantification of myocardial glucose uptake in mice. J Nucl Med. 2015;56(10):1615-1621.

17. Bax JJ, et al. Optimal metabolic conditions during fluorine-18 fluorodeoxyglucose imaging; a comparative study using different protocols. Eur J Nucl Med. 1997;24(1):35-41.

18. Bengel FM, et al. Non-invasive assessment of the effect of cardiac sympathetic innervation on metabolism of the human heart. Eur J Nucl Med. 2000;27(11):1650-1657.

19. Hernandez AM, et al. Longitudinal evaluation of left ventricular substrate metabolism, perfusion, and dysfunction in the spontaneously hypertensive rat model of hypertrophy using small-animal PET/CT imaging. J Nucl Med. 2013;54(11):1938-1945

20. Nakatani K, et al. Myocardial energy provision is preserved by increased utilization of glucose and ketone bodies in CD36 knockout mice. Metab Clin Exp. 2015;64(9):1165-1174.

21. Ouyang C, You J, Xie Z. The interplay between autophagy and apoptosis in the diabetic heart. J Mol Cell Cardiol. 2014;71:71-80

22. Guo S. Insulin signaling, resistance, and the metabolic syndrome: insights from mouse models into disease mechanisms. $J$ Endocrinol. 2014;220(2):T1-T23.

23. Connelly KA, et al. Functional, structural and molecular aspects of diastolic heart failure in the diabetic (mRen-2)27 rat. Cardiovasc Res. 2007;76(2):280-291.

24. Kolwicz SC, Purohit S, Tian R. Cardiac metabolism and its interactions with contraction, growth, and survival of cardiomyocytes. Circ Res. 2013;113(5):603-616

25. Das SK, Hoefler G. The role of triglyceride lipases in cancer associated cachexia. Trends Mol Med. 2013;19(5):292-301.

26. Abbruzzese $\mathrm{C}$, et al. Detection of phosphorylated insulin receptor in colorectal adenoma and adenocarcinoma: implications for prognosis and clinical outcome. J Cell Physiol. 2015;230(3):562-567.

27. Lundholm K, et al. Insulin treatment in cancer cachexia: effects on survival, metabolism, and physical functioning. Clin Cancer Res. 2007;13(9):2699-2706.

28. Morton DB, Griffiths PH. Guidelines on the recognition of pain, distress and discomfort in experimental animals and an hypothesis for assessment. Vet Rec. 1985;116(16):431-436.

29. Hoch M, et al. Erythropoietin preserves the endothelial differentiation capacity of cardiac progenitor cells and reduces heart failure during anticancer therapies. Cell Stem Cell. 2011;9(2):131-143.

30. Thackeray JT, Bankstahl JP, Wang Y, Wollert KC, Bengel FM. Clinically relevant strategies for lowering cardiomyocyte glucose uptake for 18F-FDG imaging of myocardial inflammation in mice. Eur J Nucl Med Mol Imaging. 2015;42(5):771-780.

31. Scherr M, et al. Differential expression of miR-17 92 identifies BCL2 as a therapeutic target in BCR-ABL-positive B-lineage acute lymphoblastic leukemia. Leukemia. 2014;28(3):554-565.

32. Hilfiker-Kleiner D, et al. Continuous glycoprotein-130-mediated signal transducer and activator of transcription-3 activation promotes inflammation, left ventricular rupture, and adverse outcome in subacute myocardial infarction. Circulation. 2010;122(2):145-155

33. Halkein J, et al. MicroRNA-146a is a therapeutic target and biomarker for peripartum cardiomyopathy. J Clin Invest. 2013;123(5):2143-2154.

34. Hilfiker-Kleiner D, et al. Signal transducer and activator of transcription 3 is required for myocardial capillary growth, control of interstitial matrix deposition, and heart protection from ischemic injury. Circ Res. 2004;95(2):187-195.

35. Hilfiker-Kleiner D, et al. A cathepsin D-cleaved $16 \mathrm{kDa}$ form of prolactin mediates postpartum cardiomyopathy. Cell. 2007;128(3):589-600.

36. Stapel B, et al. Low STAT3 expression sensitizes to toxic effects of beta-adrenergic receptor stimulation in peripartum cardiomyopathy. Eur Heart J. 2017;38(5):349-361.

37. Stuck BJ, Lenski M, Böhm M, Laufs U. Metabolic switch and hypertrophy of cardiomyocytes following treatment with angiotensin II are prevented by AMP-activated protein kinase. J Biol Chem. 2008;283(47):32562-32569.

38. Paik JY, Lee KH, Ko BH, Choe YS, Choi Y, Kim BT. Nitric oxide stimulates 18F-FDG uptake in human endothelial cells through increased hexokinase activity and GLUT1 expression. J Nucl Med. 2005;46(2):365-370. 